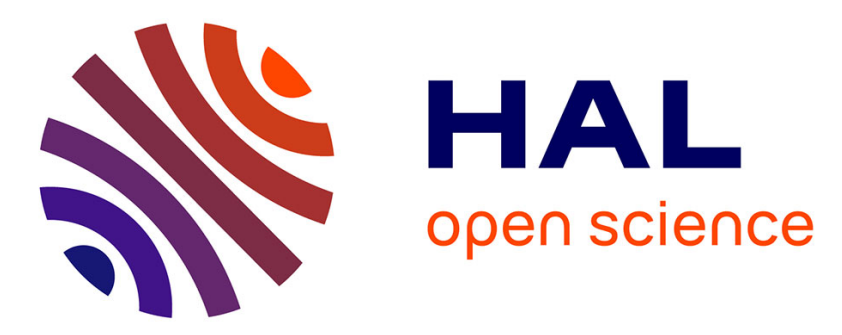

\title{
Anti-tissue factor short hairpin RNA inhibits breast cancer growth in vivo
}

\author{
J. E. Bluff, M. Amarzguioui, J. Slattery, M. W. R. Reed, N. J. Brown, C. A. \\ Staton
}

\section{- To cite this version:}

J. E. Bluff, M. Amarzguioui, J. Slattery, M. W. R. Reed, N. J. Brown, et al.. Anti-tissue factor short hairpin RNA inhibits breast cancer growth in vivo. Breast Cancer Research and Treatment, 2010, 128 (3), pp.691-701. 10.1007/s10549-010-1149-8 . hal-00615377

\section{HAL Id: hal-00615377 https://hal.science/hal-00615377}

Submitted on 19 Aug 2011

HAL is a multi-disciplinary open access archive for the deposit and dissemination of scientific research documents, whether they are published or not. The documents may come from teaching and research institutions in France or abroad, or from public or private research centers.
L'archive ouverte pluridisciplinaire HAL, est destinée au dépôt et à la diffusion de documents scientifiques de niveau recherche, publiés ou non, émanant des établissements d'enseignement et de recherche français ou étrangers, des laboratoires publics ou privés. 
Anti-Tissue Factor short hairpin RNA inhibits breast cancer growth in vivo.

JE Bluff ${ }^{1}$, M Amarzguioui ${ }^{2}$, J Slattery ${ }^{1}$, NJ Brown ${ }^{1}$, MWR Reed $^{1}$ \& CA Staton ${ }^{1 *}$.

${ }^{1}$ Academic Units of Surgical Oncology, University of Sheffield Medical School, Sheffield S10 $2 \mathrm{RX}, \mathrm{UK}$ and ${ }^{2}$ siRNAsense, University of Oslo, Norway

* Author for correspondence:

Dr Carolyn Staton,

Microcirculation Research Group,

Academic Unit of Surgical Oncology,

Department of Oncology,

Faculty of Medicine, Dentistry \& Health,

University of Sheffield

Beech Hill Road

Sheffield,

South Yorkshire,

S10 2RX, UK

tel: $+44(0) 114226-5852$

fax: +44 (0)114 271-3314

e-mail: c.a.staton@sheffield.ac.uk

Running title: TF knockdown inhibits breast cancer growth

Conflict of interest: The authors wish to declare a conflict of interest for co-author $\mathrm{Dr}$ Mohammed Amarzguioui. He serves as Research Director for the company SiRNAsense AS, which is currently developing siRNA-based drugs targeting Tissue Factor. 


\section{Abstract}

Purpose: In breast cancer there is a correlation between tissue factor (TF) expression, angiogenesis and disease progression. TF stimulates tumour angiogenesis, in part, through up-regulation of vascular endothelial growth factor (VEGF). Therefore, this study aimed to establish whether TF stimulates angiogenesis and tumour progression directly and independent of VEGF up-regulation.

Methods: Initially the effects of TF and VEGF were assessed on endothelial cell migration (Boyden chamber) and differentiation (tubule formation on Matrigel). Susbequently MDAMB-436 breast cancer cells, which produce high levels of both TF and VEGF (western blot analysis), were established in vivo, following which tumours were treated three times per week for three weeks with intra-tumoural injections of either anti-VEGF siRNA, anti-TF shRNA, the two treatments combined or relevant controls.

Results: Both VEGF and TF significantly stimulated endothelial cell migration and tubule formation $(\mathrm{P}<0.02)$. Breast cancer xenografts (MDA-MB-436) treated with TF or VEGFspecific agents demonstrated significant inhibition in tumour growth (VEGFsiRNA 61\%; final volume: $236.2+/-23.2 \mathrm{~mm}^{3}$ vs TFshRNA $89 \% ; 161.9+/-17.4 \mathrm{~mm}^{3}$ vs combination $93 \%$; $136.3+/-9.2 \mathrm{~mm}^{3}$ vs control $\left.400.4+/-32.7 \mathrm{~mm}^{3}: \mathrm{p}<0.005\right)$. Microvessel density, a measure of angiogenesis, was also significantly inhibited in all groups (MVD in control=29+/-2.9; TFshRNA $=18+/-1.1 ; \quad$ VEGFsiRNA $=16.7+/-1.5 ; \quad$ both $=12+/-2.1 ; \quad p<0.004)$, whereas the proliferative index of the tumours was only reduced in the TFshRNA treated groups (control=0.51+/-0.011; TFshRNA=0.41+/-0.014; VEGFsiRNA=0.49+/-0.013; both=0.41+/$0.004 ; p<0.008)$

Conclusions: These data suggest that TF has a direct effect on primary breast cancer growth and angiogenesis, and that specific inhibition of the TF signalling pathway has potential for the treatment of primary breast cancer. 
Key words: Angiogenesis, breast cancer, haemostasis, Tissue Factor, VEGF

Abbreviations: CCS - Cumulative Chalkley Score; GF - growth factor; HuDMEC - Human dermal microvascular endothelial cells; MVD - microvessel density; PECAM - platelet endothelial cell adhesion molecule; TF - Tissue Factor; VEGF - vascular endothelial growth factor 


\section{Introduction}

Angiogenesis, the outgrowth of new capillaries from an existing vascular bed, and haemostasis, the coagulation cascade leading to clot formation, are amongst the most consistent host responses associated with cancer. These interrelated processes have important implications for cancer therapy as hypercoagulability is exhibited by most cancer patients and contributes to the pathogenesis of tumour growth and metastasis by promoting angiogenesis [1].

Tissue factor (TF) is a $47 \mathrm{kDa}$ transmembrane glycoprotein that safeguards the vascular integrity of tissues by initiating the coagulation cascade following vessel injury [1]. In normal physiological situations, TF expression is restricted to the tunica adventitia and subendothelial cells, however during tumourigenesis, expression within vascular endothelial cells is also observed. Indeed, up-regulation of TF protein by cancer cells and associated stromal cells has been well documented in breast cancer and other malignant tumours [2-4]. TF levels have been correlated with advanced stages of malignancy and metastatic potential in different cancers, including breast [2,4], suggesting that TF may play a role in tumour progression, which may be related to the putative role of TF in metastatic disease as TF inhibition reduces both melanoma and breast cancer cell metastasis in vivo $[5,6]$.

TF indirectly contributes to tumour angiogenesis via generation of thrombin, which releases pro-angiogenic factors from activated platelets and converts circulating fibrinogen to an insoluble fibrin clot, providing a provisional matrix to facilitate blood vessel infiltration (reviewed by [7]). TF also up-regulates the pro-angiogenic protein, vascular endothelial growth factor (VEGF) [8], the most potent known stimulator of angiogenesis, and correlates with both microvessel density (MVD; a surrogate marker of angiogenesis) and VEGF expression in breast and colon cancer [2,3].

Despite increasing evidence that TF participates in many tumour-related processes, the precise role is incompletely understood, although evidence suggests that therapeutic 
targeting of TF may inhibit tumour angiogenesis and hence disease progression. This study demonstrates that TF stimulates angiogenesis independent of VEGF up-regulation and provides evidence for the first time that TF-specific knockdown using shRNA technology significantly reduces primary breast tumour growth in vivo.

\section{Methods}

\section{Cells}

Adult human dermal microvascular endothelial cells (HuDMEC; Promocell, Heidelberg), were cultured in complete microvascular endothelial cell growth medium (EGM; Promocell), and used for a maximum of 5 passages. MDA-MB-436, MCF-7 and T47D human breast cancer cell lines (ATCC) were routinely tested for key characteristics (including ER \& PR status). MDA-MB-436/MCF-7 and T47D cells were cultured in high glucose RPMI 1640 (GIBCO, Paisley, UK) and DMEM (Invitrogen) medium respectively, containing 1\% Lglutamine, 10\% FCS and 1\% penicillin-streptomycin. All cells were grown at $37 \mathrm{C}$ in a 100\% humidified incubator with a gas phase of $5 \% \mathrm{CO}_{2}$ and routinely screened for Mycoplasma.

\section{Tubule formation assay}

The HuDMEC tubule formation assay was performed as previously described [11] using EGM and $1 \%$ heat inactivated FCS containing VEGF, TF or both $(2-50 \mathrm{ng} / \mathrm{ml})$. Tubule formation was measured as the number of cord-like structures and the number of branch points seen within an entire well. Each experiment contained 3 replicate wells for each treatment and was repeated 3 times. 


\section{Migration assay}

A 48 well microchemotaxis chamber (Neuro Probe, Cabin John, MD) was used as previously described [11] with VEGF or TF alone or in combination $(2-50 \mathrm{ng} / \mathrm{ml})$ placed in the lower chamber, and serum starved HuDMEC in the top chamber. Migrated cells were then fixed, stained and counted at $\times 40$ magnification in 3 random fields per well in 3 replicate wells and repeated 3 times.

\section{Cloning of pFRT-H1 shRNA expression vectors.}

Expression plasmids pFRT-H1T-hTFsh (referred to hereafter as hTFshRNA) and pFRTH1T-mTFsh (referred to hereafter as non-targeting vector control) were generated by directional oligonucleotide cassette mediated cloning into the Bglll/Kpnl linearised expression vector pFRT-H1tetO, as previously described [9]. The shRNAs were designed to target previously identified optimal human-specific [10] and murine-specific [6] TF siRNA target sites.

The following oligonucleotides were used for cloning of the shRNA cassettes:

hTFsh-A: 5'-gatcgcgcttcaggcactacaaatactgtcctgacccaacagtatttgtagtgcctgaagcgcttttgtac-3';

hTFsh-B: 5'-aaaaagcgcttcaggcactacaaatactgttgggtcaggacagtatttgtagtgcctgaagcgc-3';

mTFsh-A: 5'-gatcgcattccagagaaagcgtttaatttcctgacccaaaattaaacgctttctctggaatgcttttgtac-3';

mTFsh-B: 5'-aaaaagcattccagagaaagcgtttaattttgggtcaggaaattaaacgcttctctggaatgc-3'

The oligos were annealed pairwise, phosphorylated, and ligated into complementary Bglll/Kpnl-linearised vector, as previously described [9]. Correct sequence of restrictionpositive transformants was confirmed by sequencing in both directions, using the primers 5'caaggtcgggcaggaagag-3' and 5'-aggggcaaacaacagatggc-3'. 


\section{VEGFSIRNA}

A VEGF Stealth ${ }^{T M}$ Select 3 RNAi pre-validated siRNA set (including three 25bp duplexes targeting non-overlapping sequences specific to VEGF) and Stealth $\mathrm{RNAi}^{\mathrm{TM}}$ siRNA negative control kit were purchased from Invitrogen Ltd (Renfrew, UK). The latter had no known homology to the target sequence. The target sequences of the VEGF siRNAs were:

\begin{tabular}{|c|c|c|}
\hline Name and bases & Strand & Sequence \\
\hline Stealth $^{\mathrm{IM}} 1$ (bases 1227-1251) & Sense & 5'-ccugaugagaucgaguacaucuuc-3' \\
\hline Stealth $^{\mathrm{TM}} 1$ (bases 1227-1251) & Anti-sense & 5'-ugaagauguacucgaucucaucagg-3' \\
\hline Stealth $^{\mathrm{TM}} 2$ (bases 1376-1400) & Sense & 5'-gcacauaggagagaugagcuucuua-3' \\
\hline Stealth $^{\mathrm{TM}} 2$ (bases 1376-1400) & Anti-sense & 5'-uaggaagcucaucucuccuaugugc-3' \\
\hline Stealth $^{\mathrm{TM}} 3$ (bases 1405-1429) & Sense & 5'-acaacaaaugugaaugcagaccaaa-3' \\
\hline Stealth $^{\mathrm{TM}} 3$ (bases 1405-1429) & Anti-sense & 5'-uuuggucugcauucacauuuguugu-3' \\
\hline
\end{tabular}

\section{Quantitative Real Time PCR (qRT-PCR)}

The mRNA was extracted using the tri-reagent system (Sigma, Dorset, UK), from directly lysed cells, and the concentration and purity of total RNA determined by UV light absorption. cDNA was synthesised from $1 \mathrm{~g}$ total RNA using the Taqman reverse transcriptase reagents (Applied Biosystems, Foster City, CA, USA) and SuperScript ${ }^{\circledR}$ II Reverse Transcriptase (Invitrogen). qRT-PCR was performed using PCR Master Mix (Eurogentec Ltd, Hampshire, UK), with predesigned sequence specific Taqman gene probes (Applied Biosystems). qRT-PCR cycling conditions were 2 minutes at $50 \mathrm{C}$ and then $95 \mathrm{C}$ for 10 minutes, followed by 40 cycles of 15 seconds at $95 \mathrm{C}$, followed by 1 minute at $60 \mathrm{C}$. qRT- 
PCR was detected by ABI-Prism 7900HT Sequence Detection System and data processed using GeneAmp (Applied Biosystems). Target gene expression (VEGF and TF mRNA) was normalised to levels of GAPDH endogenous control.

\section{Protein analysis}

Protein extracts were prepared by lysing breast cancer cells with a triple cell lysis buffer [11]. Protein concentrations were determined using the bicinchoninic acid (BCA) assay. Equal amounts of protein extracts $(20 \mathrm{~g})$ were separated via SDS polyacrylamide gel electrophoresis (PAGE), electrotransferred to nitrocellulose membranes and exposed to anti-TF (4509; American Diagnostica Inc. Connecticut, USA; $2 \mathrm{~g} / \mathrm{ml}$ ) or anti-VEGF (A-20; Santa Cruz Biotechnology, Wiltshire, UK; $5 \mathrm{~g} / \mathrm{ml}$ ). Immunoreactive bands were detected by enhanced chemiluminescence (Amersham), with -actin as a loading control (Sigma).

Alternatively, conditioned media was collected from relevant samples and centrifuged before TF and VEGF were measured in duplicate using commercial two-antibody sandwich assays kits (TF: Immubind, American Diagnostica; VEGF: Quantikine, R\&D Systems, Abingdon, Oxfordshire, UK). The detection limits of the TF and VEGF assays were $10 \mathrm{pg} / \mathrm{ml}$ and $30 \mathrm{pg} / \mathrm{ml}$, respectively.

\section{Transfections and flow cytometry}

MDA-MB-436 cells were seeded in six-well plates and grown to $40 \%$ confluence before cotransfection with Block-IT fluorescent (fluorescein [FITC]) oligo (Invitrogen) and hTFshRNA vector targeting human TF (1.2 g/well) under serum-free conditions in Opti-MEM-1 (Invitrogen). Untransfected, mock transfected (MT) (Block-IT fluorescent oligo) and cells transfected with a non-targeting vector (pFRT-H1T-mTFsh) served as controls. All experiments were performed in triplicate and repeated at least 3 times. Flow cytometry 
(FACSAria $^{\text {TM }}$ [BD Biosciences, San Jose, CA, USA]) was used to select transfected cells based on FITC expression. Transfected cells were plated and cell proliferation and viability measured using a Vi-cell counter at 2 and 4 days post transfection. RNA was also extracted at 2 and 4 days post transfection for qRT-PCR analysis and conditioned media was collected at 4 days post transfection for protein analysis via ELISA.

\section{In vivo tumourigenicity assay}

Animal experiments were conducted in accordance with the Home Office License Authority of the United Kingdom Animals (Scientific procedures) Act 1986 (PPL 40/2972; holder NJB) and with local research ethics committee approval. MDA-MB-436 cells $\left(1 \times 10^{7}\right)$ were grown subcutaneously in athymic nude mice until the tumour reached approximately $100 \mathrm{~mm}^{3}$ (range $=104-119 \mathrm{~mm}^{3}$, mean $+/-\mathrm{SE}=108.9+/-3.2$ ). Mice were randomly assigned to treatment groups (6/group) and received intra-tumoural injections, 3 times per week for 3 weeks, of either anti-VEGFsiRNA (6 g in 20 I lipofectamine 2000; Invitrogen), or antiTFshRNA (10 $\mu \mathrm{g}$ in 20 I lipofectamine LTX; Invitrogen), or the two treatments combined; doses were based on previous studies with siRNA [12] or shRNA [13]. Another group of mice received no treatment (WT), while a non-targeting shRNA vector and a scrambled (non-targeting) siRNA were used as negative controls. Tumour growth was assessed using calipers every $2 / 3$ days. At the end of the study tumours and control tissues were excised and bisected for immunohistochemical analysis.

\section{Immunohistochemical staining}

Tissues were fixed in either $10 \%$ neutral buffered formalin or zinc-based fixative for 48 hours, then processed into paraffin wax. Immunohistochemistry was performed using a panel of antibodies to murine platelet endothelial cell adhesion molecule-1 (PECAM-1/CD31; 
1:100 using zinc-fixed tissues) (Pharmingen, San Diego, CA, USA), human Ki67 (1:100; Dakocytomation Ltd, Cambridgeshire, UK), VEGF (A-20; 1:150; Santa-Cruz Biotechnology Inc. Santa Cruz, CA, USA), TF (1:150; R\&D Systems) using a standard horse radish peroxidase staining procedure [14]. Negative controls were achieved by omission of the primary antibody and replacement with normal mouse IgG (DAKO), normal goat IgG (DAKO) or normal rabbit $\operatorname{lgG}(\mathrm{DAKO})$ at a dilution of 1:100.

\section{Histological analysis}

Formalin-fixed tissue sections were stained using Haematoxylin \& Eosin and tumour necrosis was assessed using a Chalkley grid method [15]. Microvascular density (MVD), a surrogate marker of angiogenesis, was assessed by obtaining a Chalkley grid score for 5 hotspots per section and the sum of the Chalkley scores for each section was termed the cumulative Chalkley score (CSS) [16]. Ki67 staining was assessed as the percentage of tumour cells showing positive staining for Ki67 antigen by counting a minimum of 1000 cells per tumour section in a total of four areas of viable tumour per section [17].

\section{Statistical analysis}

Statistical analysis was conducted using SPSS v16 software (Chicago, IL, USA). All data presented are means $+/$ - SEM, and representative data from one of 3 replicate experiments are shown. Statistical analysis was performed using a one-way analysis of variance (ANOVA) and either the Dunnet's or Mann-Whitney $U$ post hoc test for nonparametric data. Data were considered statistically significant at $p<0.05$.

\section{Results}

\section{Effects of TF on HuDMECs in vitro}


Tubule formation: HuDMECs elongated and formed tubules on GF reduced Matrigel in the absence of exogenous stimuli (due to the residual level of growth factors remaining in this matrix), but this was significantly enhanced with the addition of VEGF $(p<0.01)$ or to a lesser extent by TF $(p<0.02)$ as assessed by the number of tubules and branch points/well (Table 1, Figure $1 \mathrm{~A})$. The effects of TF on tubule formation were not attributable to stimulation of VEGF production by HuDMECs as an ELISA showed no change in secreted VEGF levels in response to $2-50 \mathrm{ng} / \mathrm{ml}$ TF concentrations (untreated $=31+/-3.2 \mathrm{pg} / \mathrm{ml}$, TF treated=28+/-4.5 $\mathrm{pg} / \mathrm{ml}$ ). When added in combination there was a non-significant increase in tubule number and no difference in the number of branch points compared to either factor alone (Figure 1).

Migration: HuDMECs migrated across the fibronectin coated filters in the absence of exogenous stimuli, however this was significantly increased in the presence of VEGF $(p<0.007)$ or TF $(p<0.001)$ (Table 2$)$. Moreover, VEGF stimulated TF to a significantly greater extent than TF $(p<0.01)$ and the combination of both factors enhanced migration more than either factor alone, although this failed to reach significance (Figure 2).

\section{Effect of p-FRT-H1-TFShRNA on TF/VEGF expression}

In order to identify a breast cancer cell line that expressed both TF and VEGF for subsequent study, qRT-PCR and western blot analysis was used to determine basal TF and VEGF gene and protein expression in MDA-MB-436, MCF-7 and T47D breast cancer cell lines. VEGF mRNA was detected in all cell lines examined (Figure 3A). In contrast, only MDA-MB-436 cells expressed TF mRNA (higher than VEGF mRNA levels), with negligible TF mRNA detected in either MCF-7 or T47D cells. Western blot analysis was consistent with the gene expression data, showing VEGF expressed by all cell lines, but only detectable levels of TF expressed in MDA-MB-436 cells (Figure 3B).

The effects of TF knockdown on TF/VEGF gene and protein expression in MDA-MB436 cells following transfection with a TF-specific shRNA vector were then determined. 
Transfection with hTFshRNA significantly decreased both TF mRNA $(77+/-3.3 \% ; p<0.001)$ and protein release $(80.3+/-3.7 \% ; p<0.003)$ (Figure $3 C \& D$ ), whereas VEGF gene expression was unaffected by TF knockdown. Furthermore, there was no difference in VEGF/TF mRNA and protein expression between cells in all control conditions. TF knockdown in MDA-MB-436 cells had no significant effect on proliferation or cell viability assessed using a Vi-cell counter over 4 days (data not shown). Essentially similar results were obtained using an alternative TF expressing breast cancer cell line, MDA-MB-231, showing 75\% knockdown in expression.

Intra-tumoural injections of anti-TFshRNA and anti-VEGFsiRNA inhibits tumor growth in vivo

Direct intra-tumoural injections of anti-TFshRNA or anti-VEGFsiRNA alone and in combination, were performed on established MDA-MB-436 tumours to determine whether TF expression plays a key role in primary breast tumour growth and angiogenesis that is functionally distinct from the role in VEGF production (Figure 4). Importantly, the nontargeting vector and scrambled siRNA control treatments had no significant effect on final tumour volume compared to the untreated group $(367.2+/-35.9,352.1+/-23.1$ and $400.4+/-$ $32.7 \mathrm{~mm}^{3}$ respectively at day 21$)$. In contrast, the TF or VEGF-specific treatments all significantly $(p<0.005)$ inhibited tumour growth; treatment with anti-VEGFsiRNA by $61 \%$ (final volume: $236.2+/-23.2 \mathrm{~mm}^{3}$ ) or TFshRNA by $89 \%\left(161.9+/-17.4 \mathrm{~mm}^{3}\right)$ alone, while the combination resulted in $93 \%$ growth inhibition $\left(136.3+/-9.2 \mathrm{~mm}^{3}\right)$. Interestingly the tumour growth inhibition induced by TFshRNA alone or in combination with VEGFsiRNA was significantly greater than treatment with VEGFsiRNA alone $(p=0.018$ and $p=0.016$ respectively; Figure 4). 
Histological analysis of control tumour tissue revealed the presence of a central area of necrosis surrounded by a viable rim of tumour cells, which was unchanged any of the treatment regimes (data not shown). To determine whether the reduced volume of both TFshRNA and VEGFsiRNA treated tumours was due to an effect on the tumour vasculature, endothelial cells were identified using CD31 staining (Figure 5A). Microvessel density in the viable rim of tumour cells was significantly reduced compared to relevant controls in VEGFsiRNA $(p=0.002)$, TFshRNA $(p=0.002)$ and combined $(p=0.004)$ treatment groups (Figure 5A). In order to determine whether the reduced tumour volume was also due to an effect on tumour cell proliferation, the proliferation index was calculated on Ki67 stained sections [17]. In contrast to MVD, only TFshRNA treatment had a significant effect on proliferation index (control: $0.51+/-0.01$ vs TFshRNA: $0.41+/-0.02 ; p=0.008$, Figure $5 B$ ). VEGF staining revealed a relatively homogenous pattern throughout the cytoplasm of tumour cells, with the intensity of staining reduced, but not completely absent in the VEGFsiRNA and combined treated tumours only (Online Resource 1A). In contrast, TF staining was seen in the membrane of tumour cells and was heterogeneous, with more intense staining adjacent to the necrotic regions of the tumours. TFshRNA treated tumours showed less staining than any other tumours and contained some areas where TF staining was absent (Online Resource 1B).

\section{Discussion}

Elevated TF expression in breast cancer has been associated with disease progression, poor survival, increased VEGF expression and angiogenesis [2, 4]. VEGF is known to up-regulate the expression of TF in cancer cells and associated endothelial cells, potentially creating a feedback loop whereby TF in turn up-regulates VEGF expression [1]. It has therefore been proposed that inhibition of TF in breast cancer may result in reduced angiogenesis due to down-regulation of VEGF expression. The data presented here 
however, provide evidence that TF has the ability to modulate angiogenic mechanisms in vitro in the absence of VEGF up-regulation. Moreover, we demonstrate for the first time that direct intra-tumoural injections of TFshRNA prevents the growth of established primary breast cancer xenografts in vivo by inhibiting both tumour cell proliferation and angiogenesis.

It has been well documented that VEGF stimulates angiogenic activities in endothelial cells (reviewed in [18]) and our data is consistent with other studies showing a dose-dependent increase in HuDMEC migration and tubule formation in vitro. TF also stimulates HuDMEC migration and tubule formation/differentiation, although to a lesser extent, and appears not to be dose dependent or resulting from VEGF up-regulation. This is in agreement with studies demonstrating that soluble TF promotes EC migration and tubule formation [19] and that an alternatively spliced form of TF (asTF) induces tubule formation independently of VEGF or PAR signaling [20]. In vitro assays from the current study were carried out in the absence of activated factor VII (FVIIa), by using low levels of heat inactivated serum, and data indicate a direct role for TF signaling in EC migration and tubule formation, although the precise mechanisms mediating this response are as yet undetermined. Interestingly, there was only a small additional increase in tubule formation or migration in the presence of both TF and VEGF, compared to VEGF alone, suggesting that VEGF, the most potent angiogenic factor identified to date [8], is likely to be saturating the endothelial cell activation and functional responses in these assays.

In breast cancer, TF is associated with metastatic potential and poor prognosis [4] in addition to playing a role in angiogenesis [2]. The current study demonstrates that TF is highly expressed in metastatic breast carcinoma cells (MDA-MB-436), in contrast to nonmetastatic breast carcinoma cells (MCF-7, T47D), although MCF-7 expresses TF under hypoxic conditions (data not shown; [21]). These observations are in agreement with previous studies, reporting elevated TF expression in metastatic cell lines compared to nonmetastatic cell lines of the same tissue origin [22-24]. Following TF expression knockdown of $\sim 80 \%$ in the MDA-MB-436 cell line there was no effect on VEGF expression, suggesting that 
TF is not involved in regulating VEGF expression in this cell line. Furthermore, TF knockdown had no effect on cell proliferation or viability in vitro over a time course of 4 days, in agreement with other studies using a similar mouse specific construct in B16 melanoma cells [6].

As previous studies had investigated the effects of murine-specific TF shRNA on metastasis [6], we used a model of established primary MDA-MB-436 subcutaneous tumours treated with intra-tumoural injections of TFshRNA or VEGFsiRNA alone and in combination, to achieve direct homology-dependent post-transcriptional gene silencing, with subsequent knockdown of protein translation. Our data show that VEGF inhibition significantly reduces primary breast tumour growth and angiogenesis (measured by MVD), in agreement with results from relevant mouse xenograft studies [25-26, 12]. Interestingly, treatments with TFshRNA (either alone or in combination with VEGFsiRNA) were more potent in reducing primary tumour growth than VEGFsiRNA treatment alone. However, shRNA requires cellular transcription in the nucleus, further processing, then transport to the cytoplasm before being incorporated into the RNA-interfering silencing complex, therefore data generated from administration of siRNA and shRNA are not directly comparable. What is interesting is that addition of TFshRNA to VEGFsiRNA significantly increases tumour inhibition compared to VEGFsiRNA alone. Moreover, these data suggest that TF has a direct role in promoting primary tumour growth that is functionally distinct from the role in VEGF production. This is in agreement with previous in vivo studies using stable TF knockdown in colorectal cancer cells grown in Matrigel plugs which showed a reduction in angiogenesis, but only minimal effects on VEGF expression [27]. Although other groups have previously shown that inhibition of TF signaling suppresses hypoxia-induced retinal angiogenesis [28] and breast tumour growth in vivo [5, 29], this is the first study to evaluate TF inhibition alone and in combination with VEGF inhibition, in established primary breast tumours. 
Our in vivo data show that both treatments (TF and VEGF knockdown) inhibit angiogenesis, but only tumours treated with TFshRNA showed a significantly reduced tumour proliferative index, suggesting that TF knockdown in breast tumour cells reduces proliferation in vivo, in addition to inhibiting angiogenesis. These data therefore indicate that down-regulation of VEGF expression can inhibit co-option or invasion of murine ECs, whilst the anti-tumour response to TF knockdown may also, in part, be attributed to direct effects on breast tumour cell proliferation. This data is consistent with observations from previous studies on gene expression in a human melanoma cell line demonstrating down-regulation of genes involved in cell growth, invasion and metastasis and an up-regulation of genes involved in apoptosis, and reduced cell proliferation following TF knockdown using TFshRNA [10]. Differences between in vitro and in vivo proliferation responses are likely to be attributable to other tumour microenvironmental factors in vivo. Although breast cancer cell lines express PAR-2, which becomes activated in the presence of TF-FVIla stimulating signaling pathways potentially resulting in proliferation [30], data relating to the direct TF effects on proliferation are contradictory [27,31-32].

Whilst the current study using TFshRNA, and others using inactivating peptides and antibodies [5, 29], show a definite effect of TF inhibition on primary tumour growth in vivo, clinical applications of systemic anti-TF therapy are likely to be associated with significant risks, due to the essential role of the haemostatic pathway. Indeed, a phase I clinical trial using a monoclonal antibody against TF showed an increase in the frequency of haemorrhagic events such as oral mucosal bleeding, cutaneous bleeding and spontaneous tongue haematomas in a dose dependent manner [33]. These side effects may be circumvented by the development of specific inhibitors that suppress TF-FVIla signaling in pathological angiogenesis, but not in TF-FVIla coagulant function [29], although such an approach requires full evaluation. Alternative approaches such as using atellocollagen or nanoparticles to specifically target shRNA/siRNAs to tumours following systemic administration, are also beginning to show some efficacy in vivo [34-36]. These methods 
require further validation, but may prove effective for tumour-specific targeting of TF with shRNA or siRNA via systemic delivery, thereby circumventing the haemostatic side-effects associated with systemic anti-TF treatment.

In conclusion, these data suggest for the first time that modulation of TF has a direct effect on established primary breast cancer growth and angiogenesis that is distinct from the role in VEGF production. Therefore, similar to a potential use suggested for gliomas [32], specific inhibition of the TF signaling pathway with shRNA or siRNA represents a powerful tool for inhibiting cancer cell proliferation and angiogenesis in vivo with potential therapeutic utility for the effective treatment of primary breast cancers using targeted therapy. Further work is now required to investigate the effects of TF knockdown in models relevant to breast cancer metastasis.

Acknowledgements: We gratefully acknowledge the technical support of Jenny Globe, Carmel Nichols and Yvonne Stephenson. This work was supported by Yorkshire Cancer Research (grant number S287).

\section{References:}

1. Bluff JE, Brown NJ, Reed MWR, Staton CA (2008) Tissue Factor, angiogenesis and tumour progression (review). Breast Cancer Research 10:204-214.

2. Bluff JE, Menakuru SR, Cross SS, Higham SE, Balasubramanian SP, Brown NJ, Reed MW, Staton CA (2009) Angiogenesis is associated with hyperplasia in human ductal breast disease. Br J Cancer 101:666-672.

3. Staton CA, Chetwood AS, Cameron IC, Cross SS, Brown NJ, Reed MWR (2007) The angiogenic switch occurs at the adenoma stage of the adenoma carcinoma sequence in colorectal cancer. Gut 56:1426-1432.

4. Ueno T, Toi M, Koike M, Nakamura S, Tominaga T (2000) Tissue factor expression in 
breast cancer tissues: its correlation with prognosis and plasma concentration. $\mathrm{Br} \mathrm{J}$ Cancer 83:164-170.

5. Ngo CV, Picha K, McCabe F, et al (2007) CNTO 859, a humanized anti-tissue factor monoclonal antibody, is a potent inhibitor of breast cancer metastasis and tumor growth in xenograft models. Int J Cancer 120:1261-1267.

6. Amarzguioui M, Peng Q, Wiiger MT, et al (2006) Ex vivo and in vivo delivery of anti-Tissue Factor siRNA inhibits mouse pulmonary metastasis of B16 melanoma cells. Clin Cancer Research 12:4055-4061.

7. Rak J, Milsom C, May L, Klement P, Yu J (2006) Tissue factor in cancer and angiogenesis: the molecular link between genetic tumor progression, tumour neovascularization, and cancer coagulopathy. Semin Thromb Hemost 32: 54-70.

8. Abe K, Shoji M, Chen J, et al (1999) Regulation of vascular endothelial growth factor production and angiogenesis by the cytoplasmic tail of tissue factor. Proc Natl Acad Sci USA 96:8663-8668.

9. Aagaard L, Amarzguioui M, Sun G, et al (2007) A facile lentiviral vector system for expression of doxycycline-inducible shRNAs: knockdown of the pre-miRNA processing enzyme Drosha. Mol Ther 15:938-945.

10. Wang X, Wang M, Amarzguioui M, Liu F, Fodstad Ø, Prydz H (2004) Down-regulation of Tissue Factor by RNA interference in human melanoma LOX-L cells reduce pulmonary metastasis in nude mice. Int J Cancer 112:994-1002.

11. Krajewska E, Lewis CE, Tazzyman S, Chen Y-Y, Staton CA (2010) A novel fragment derived from the beta chain of human fibrinogen, 43-63, is a potent inhibitor of activated endothelial cells in vitro and in vivo. Br J Cancer 102:594-601

12. Filleur S, Courtin A, Ait-Si-Ali S, et al (2003) SiRNA-mediated inhibition of vascular endothelial growth factor severely limits tumor resistance to antiangiogenic thrombospondin- 
1 and slows tumor vascularization and growth. Cancer Res 63:3919-3922.

13. Lin X, Yu Y, Zhao H, Zhang Y, Manela J, Tonetti DA (2006) Overexpresion of PKC is required to impart estradiol inhibition and tamoxifen-resistance in a T47D human breast cancer tumour model. Carcinogenesis 27:1538-1546.

14. Staton CA, Stribbling SM, Garcia-Echeverria C, Bury JP, Tazzyman S, Lewis CE, Brown NJ (2007) Identification of key residues involved in mediating the in vivo anti-tumour/antiendothelial activity of Alphastatin. J Thromb Haemost 5:846-54

15. Tozer GM, Bhujwalla ZM, Griffiths JR, Maxwell RJ (1989) Phosphorus-31 magnetic resonance spectroscopy and blood perfusion of the RIF-1 tumor following X-irradiation. Int $\mathrm{J}$ Radiat Oncol Biol Phys 16:155-164

16. Staton CA, Brown NJ, Rodgers GR, Corke KP, Tazzyman S, Underwood JCE, Lewis CE (2004) Alphastatin, a 24 amino-acid fragment of human fibrinogen, is a potent new inhibitor of activated endothelial cells in vitro and in vivo. Blood 103: 601-606

17. Morrone FB, Oliveira DL, Gamermann $\mathrm{P}$, et al (2006) In vivo glioblastoma growth is reduced by apyrase activity in a rat glioma model. BMC Cancer 6:226

18. Ferrara N, Gerber HP, LeCouter J (2003) The biology of VEGF and its receptors. Nat Med 9:669-676.

19. He Y, Chang G, Zhan S, Song X, Wang X, Luo Y (2008) Soluble tissue factor has unique angiogenic activities that selectively promote migration and differentiation but not proliferation of endothelial cells. Biochem Biophys Res Commun 370:489-494.

20. Hobbs JE, Zakarija A, Cundiff DL, et al (2007) Alternatively spliced human tissue factor promotes tumor growth and angiogenesis in a pancreatic cancer tumor model. Thromb Res 120:S13-S21

21. Amirkhosravi A, Meyer T, Warnes G et al (1998) Pentoxifylline inhibits hypoxia-induced upregulation of tumor cell tissue factor and vascular endothelial growth factor Thromb 
Haemost 80:598-602

22. Sawada M, Miyake S, Ohdama S, et al (1999) Expression of tissue factor in non-smallcell lung cancers and its relationship to metastasis. Br J Cancer 79:472-477.

23. Kataoka $\mathrm{H}$, Uchino $\mathrm{H}$, Asada $\mathrm{Y}$, et al (1997) Analysis of tissue factor and tissue factor pathway inhibitor expression in human colorectal carcinoma cell lines and metastatic sublines to the liver. Int J Cancer 72:878-884.

24. Mueller BM, Reisfeld RA, Edgington TS, Ruf W (1992) Expression of tissue factor by melanoma cells promotes efficient hematogenous metastasis. Proc Natl Acad Sci USA 89:11832-11836.

25. Matsumoto G, Kushibiki T, Kinoshita Y, et al (2006) Cationized gelatin delivery of a plasmid DNA expressing small interference RNA for VEGF inhibits murine squamous cell carcinoma. Cancer Sci 97:313-321.

26. Tao J, Tu YT, Huang CZ, et al (2005) Inhibiting the growth of malignant melanoma by blocking the expression of vascular endothelial growth factor using an RNA interference approach. Br J Dermatol 153:715-724.

27. Yu JL, May L, Lhotak V, et al (2005) Oncogenic events regulate tissue factor expression in colorectal cancer cells: implications for tumor progression and angiogenesis. Blood 105:1734-1741.

28. Uusitalo-Jarvinen H, Kurokawa T, Mueller BM, et al (2007) Role of protease activated receptor 1 and 2 signaling in hypoxia-induced angiogenesis. Arterioscler Thromb Vasc Biol 27:1456-1462.

29. Versteeg HH, Schaffner F, Kerver M, et al (2008) Inhibition of tissue factor signaling suppresses tumor growth. Blood 111:190-199.

30. Morris DR, Ding Y, Ricks TK, Gullapalli A, Wolfe BL, Trejo J (2006) Protease-activated receptor-2 is essential for factor VIla and Xa-induced signaling, migration, and invasion of 
breast cancer cells. Cancer Res 66:307-314.

31. Zhang $Y$, Deng $Y$, Luther $T$, et al (1994) Tissue factor controls the balance of angiogenic and antiangiogenic properties of tumor cells in mice. J Clin Invest 94:1320-1327.

32. Ge ler F, Voss V, Dutzmann S, Gerlach R, Kogel D (2010) Inhibition of tissue factor/protease-activated receptor-2 signaling limits proliferation, migration and invasion of malignant glioma cells. Neuroscience 165:1312-22

33. Morrow DA, Murphy SA, McCabe CH, Mackman N, Wong HC, Antman EM (2005) Potent inhibition of thrombin with a monoclonal antibody against tissue factor (Sunol-cH36): results of the PROXIMATE-TIMI 27 trial. Eur Heart $\mathrm{J}$ 26:682-688.

34. Kim SH, Jeong JH, Lee SH, Kim SW, Park TG (2008) Local and systemic delivery of VEGF siRNA using polyelectrolyte complex micelles for effective treatment of cancer. J Control Release 129:107-116.

35. Mu P, Nagahara S, Makita N, Tarumi Y, Kadomatsu K, Takei Y (2009) Systemic delivery of siRNA specific to tumor mediated by atelocollagen: Combined therapy using siRNA targeting Bcl-xL and cisplatin against prostate cancer. Int J Cancer 125:2978-2990

36. Yagi N, Manabe I, Tottori T, et al (2009) A nanoparticle system specifically designed to deliver short interfering RNA inhibits tumor growth in vivo. Cancer Res 69:6531-6538

\section{Figure Legends}

Figure 1: Tubule formation in response to VEGF and TF. (A) the number of tubes and (B) the number of branch points formed in response to VEGF, TF or both at $10 \mathrm{ng} / \mathrm{ml}$ or $20 \mathrm{ng} / \mathrm{ml}$. " $\mathrm{p}<0.01$ with respect to relevant control group. (C) Representative photographs in response to (I) control, (II) $10 \mathrm{ng} / \mathrm{ml}$ VEGF, (III) $10 \mathrm{ng} / \mathrm{ml} \mathrm{TF}$, and (IV) $10 \mathrm{ng} / \mathrm{ml} \mathrm{VEGF}+$ $10 \mathrm{ng} / \mathrm{ml} \mathrm{TF}$. 
Figure 2: HuDMEC migration in response to VEGF and TF. (A) Graph showing effect of VEGF or TF alone or in combination on cell migration at 10 or $20 \mathrm{ng} / \mathrm{ml}$. ${ }^{*} p<0.001$ with respect to relevant control group. (B) Representative photographs of cell migration in response to (I) control, (II) $10 \mathrm{ng} / \mathrm{ml}$ VEGF, (III) $10 \mathrm{ng} / \mathrm{ml} \mathrm{TF}$, and (IV) $10 \mathrm{ng} / \mathrm{ml} \mathrm{VEGF}+$ 10ng/ml TF.

Figure 3: Endogenous VEGF and TF expression in breast cancer cell lines and TF knockdown studies. (A) qRT-PCR analysis of VEGF and TF mRNA expression $(n=3)$.

Western Blot analysis of VEGF and TF. Scans of original blots with -actin used as loading controls. (C) qRT-PCR analysis of TF and VEGF mRNA expression following transfection of MDA-MB-436 cells at 4 days post transfection. (D) ELISA analysis of TF protein released into the media by cells at 4 days post transfection. All data were normalised for total mRNA/protein concentration and presented as a percentage of non-transfected controls. (NT - non-transfected cells; MT - mock transfected cells).

Figure 4. Response of tumours to anti-VEGFsiRNA and/or anti-TFshRNA treatment. Established MDA-MB-436 subcutaneous tumours $\left(\sim 100 \mathrm{~mm}^{3}\right)$ were treated by intra-tumoural injection three times per week. Graph showing tumour volume over the time course of treatment. VEGFsiRNA, TFshRNA and the combined treatment group significantly inhibited tumour growth over the course of the experiment. ${ }^{*}=p<0.05$ and ${ }^{* *}=p<0.005$ at day 21 . WT no treatment; NC TF - non-targeting TFshRNA; NC VEGF - scrambled VEGF siRNA.

Figure 5. Histological analysis of tumours treated with anti-VEGFsiRNA and/or antiTFshRNA. (A) Graph showing the cumulative Chalkley Score (MVD) for the tumours. 
${ }^{\wedge}=\mathrm{p}<0.004$ with respect to relevant non-targeting siRNA or shRNA group (i.e. NC TF or NC VEGF). Representative photographs of tumours stained for CD31 to assess differences in vessel morphology and density (arrows indicate vessels). (B) Graph showing the proliferation index for the tumours. $+=p<0.008$ with respect to NC TF group. Representative photographs of tumours stained for Ki67 (dark grey-black nuclei) to assess proliferation index.

\section{Online Resources}

Online Resource 1. Immunohistological analysis of siRNA/shRNA treated tumours. Representative photographs of tumours stained with (A) VEGF or (B) TF. (A) VEGF staining is homogenous and slightly reduced in VEGFsiRNA treated tumours compared to controls or TFshRNA treated tumours. There is no evidence of TFshRNA treated tumours reducing VEGF expression in these tumours. (B) TF staining is heterogenous in all tumours, although there are some areas with no TF expression in the TFshRNA treated tumours $\left(^{*}\right)$. There is no evidence of VEGFsiRNA reducing TF expression in these tumours.

\begin{tabular}{|c|c|c|c|c|}
\hline \multirow{2}{*}{$\begin{array}{c}\text { Concentration } \\
(\mathbf{n g} / \mathrm{ml})\end{array}$} & $\begin{array}{c}\text { Number of } \\
\text { tubules }\end{array}$ & $\begin{array}{c}\text { Number of } \\
\text { branch points }\end{array}$ & $\begin{array}{c}\text { Number of } \\
\text { tubules }\end{array}$ & $\begin{array}{c}\text { Number of } \\
\text { branch points }\end{array}$ \\
\cline { 2 - 5 } & $45.5 \pm 1.6$ & $4.7 \pm 0.1$ & $43 \pm 3.9$ & $5.1 \pm 0.4$ \\
\hline $\mathbf{0}$ & $54.8 \pm 4.1$ & $8.1 \pm 0.8^{\star}$ & $57.1 \pm 2.6^{*}$ & $8.3 \pm 0^{*}$ \\
\hline $\mathbf{2}$ & $60.9 \pm 3.6^{*}$ & $10.2 \pm 1.2^{*}$ & $58 \pm 3.2^{*}$ & $9.8 \pm 0.7^{*}$ \\
\hline $\mathbf{5}$ & $67.3 \pm 2.1^{*}$ & $11.4 \pm 0.8^{*}$ & $61.6 \pm 2.8^{*}$ & $12.11 \pm 0.8^{\star}$ \\
\hline $\mathbf{1 0}$ & $72.2 \pm 1.5^{*}$ & $13.7 \pm 0^{*}$ & $64.5 \pm 2.8^{*}$ & $12.7 \pm 0.4^{*}$ \\
\hline $\mathbf{2 0}$ & & &
\end{tabular}


Table 1. Effect of concentration curves of VEGF or TF on HuDMEC tubule formation as measured by number of tubules or number of branch points. ${ }^{*} P<0.02$ with respect to relevant no factor control.

\begin{tabular}{|c|c|c|}
\hline Concentration $(\mathbf{n g} / \mathbf{m l})$ & VEGF & TF \\
\hline $\mathbf{0}$ & $56 \pm 4.1$ & $55.7 \pm 3.8$ \\
\hline $\mathbf{2}$ & $71.1 \pm 2.5^{\star}$ & $65.8 \pm 3.4$ \\
\hline $\mathbf{5}$ & $81.6 \pm 1.1^{*}$ & $83.2 \pm 2.2^{*}$ \\
\hline $\mathbf{1 0}$ & $94.4 \pm 0.9^{*}$ & $88.6 \pm 1.9^{*}$ \\
\hline $\mathbf{2 0}$ & $105.3 \pm 2.6^{*}$ & $87 \pm 3.1^{*}$ \\
\hline $\mathbf{5 0}$ & $96.1 \pm 1.7^{*}$ & $86.4 \pm 2.2^{*}$ \\
\hline
\end{tabular}

Table 2. Effect of concentration curves of VEGF or TF on HuDMEC migration. ${ }^{*} \mathrm{P}<0.007$ with respect to relevant no factor control. 

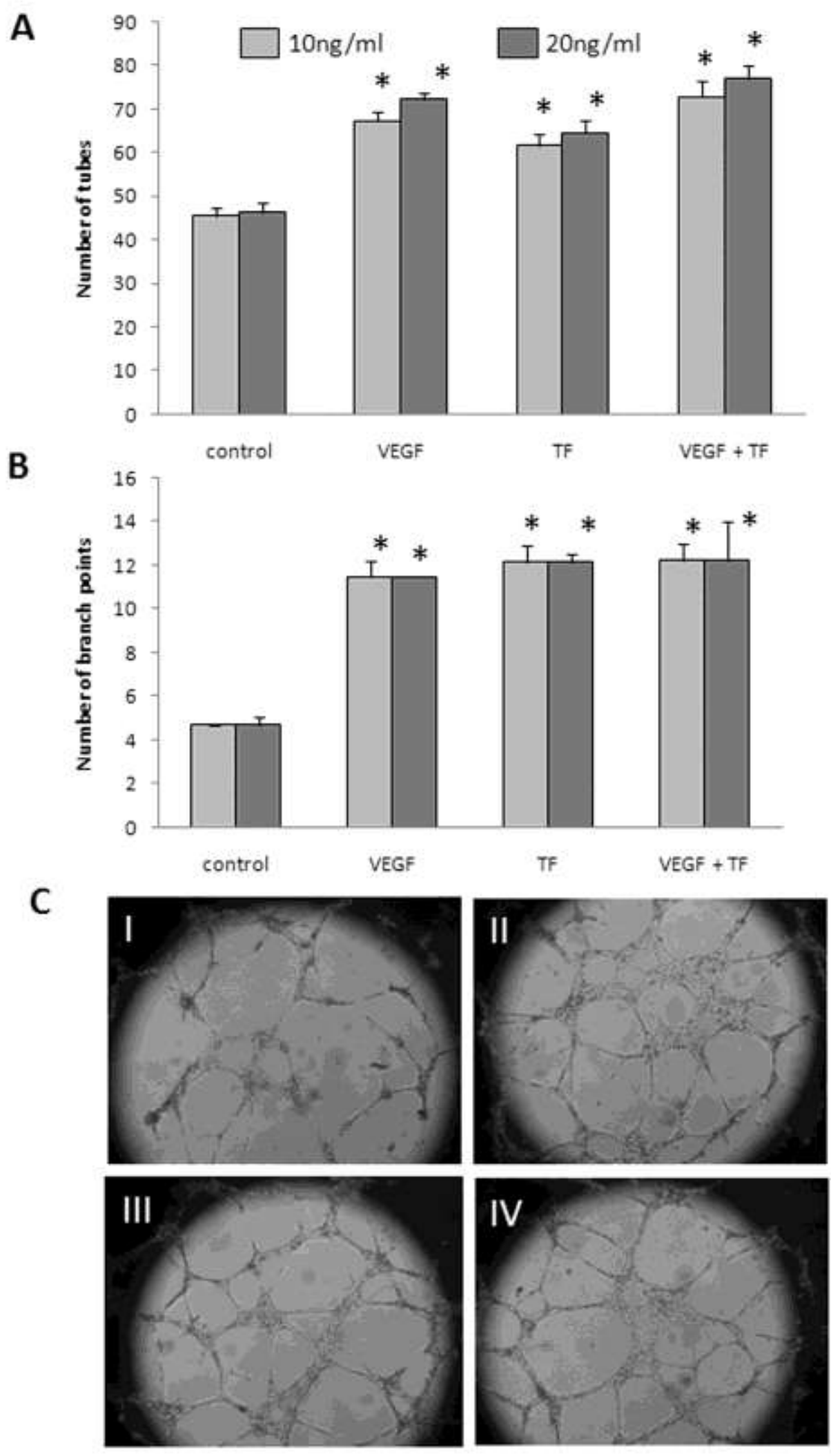

Figure 1 
A

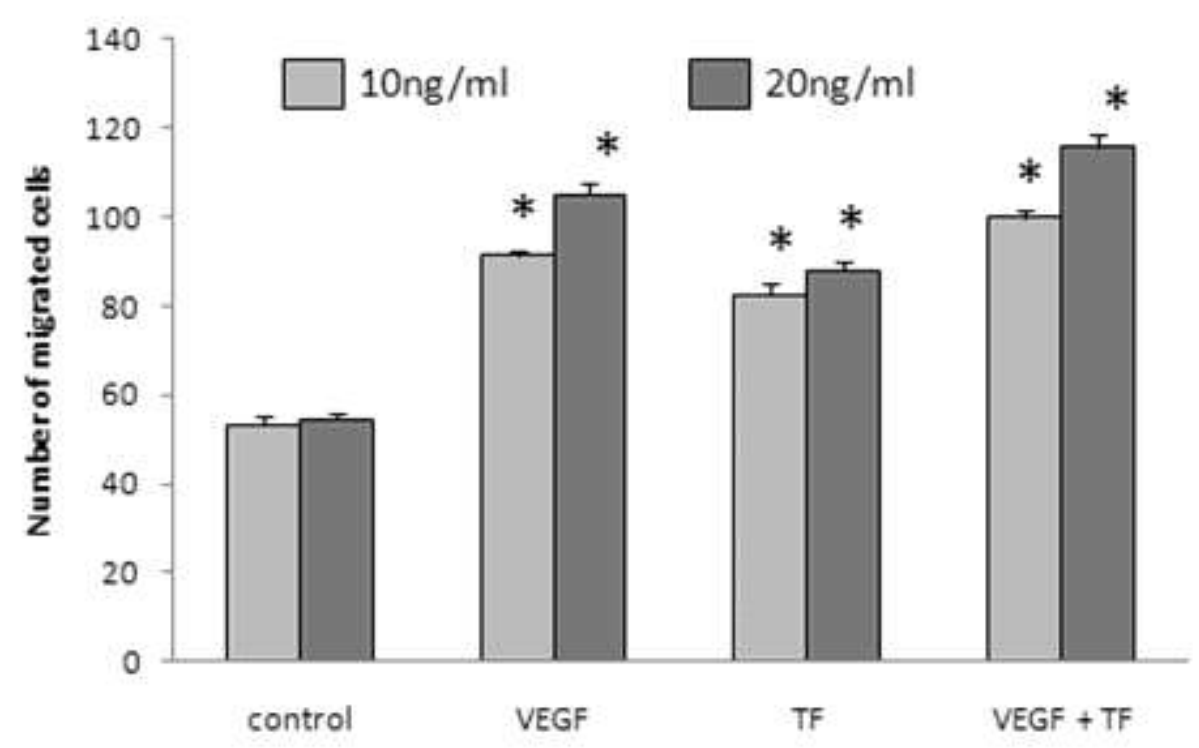

B
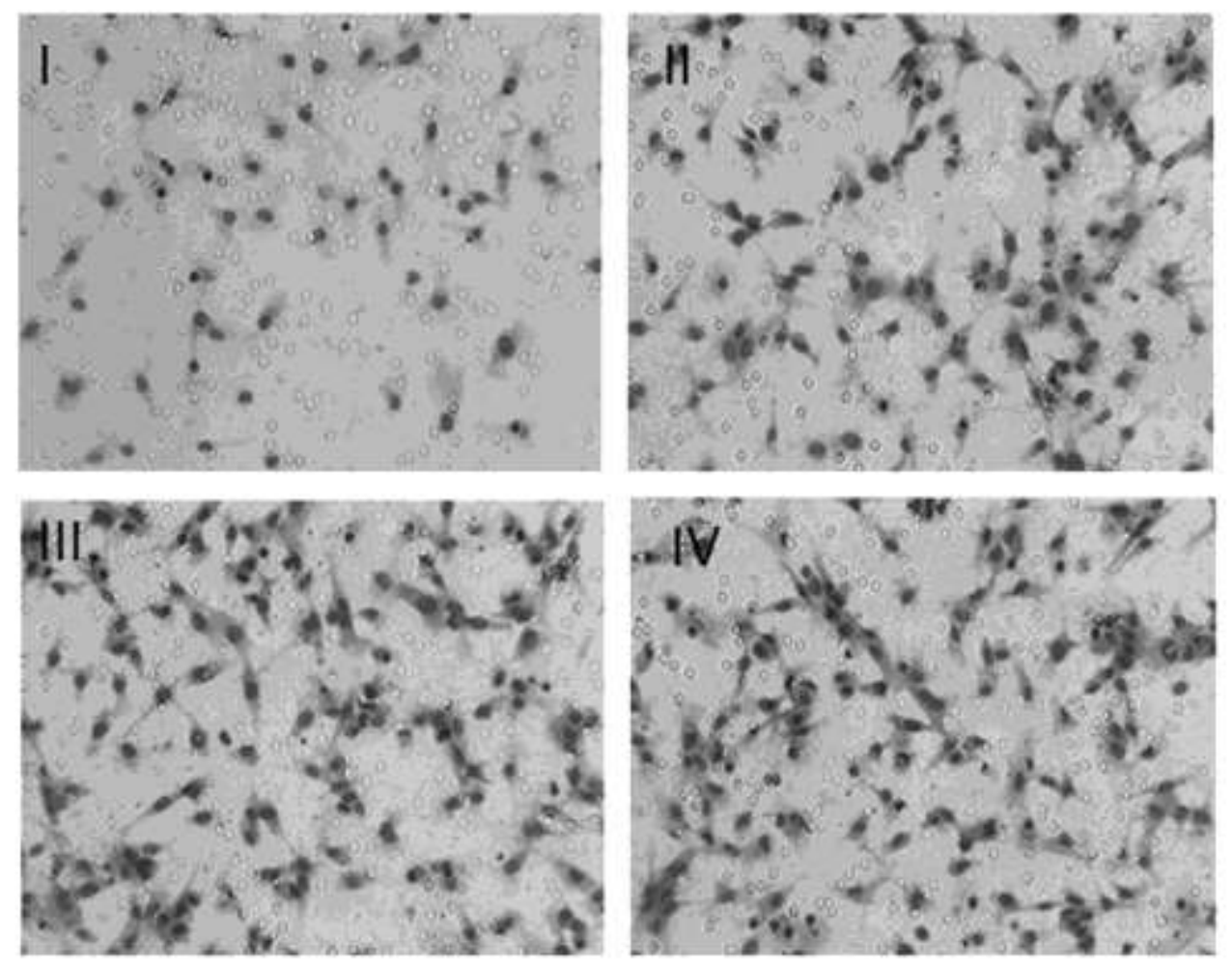

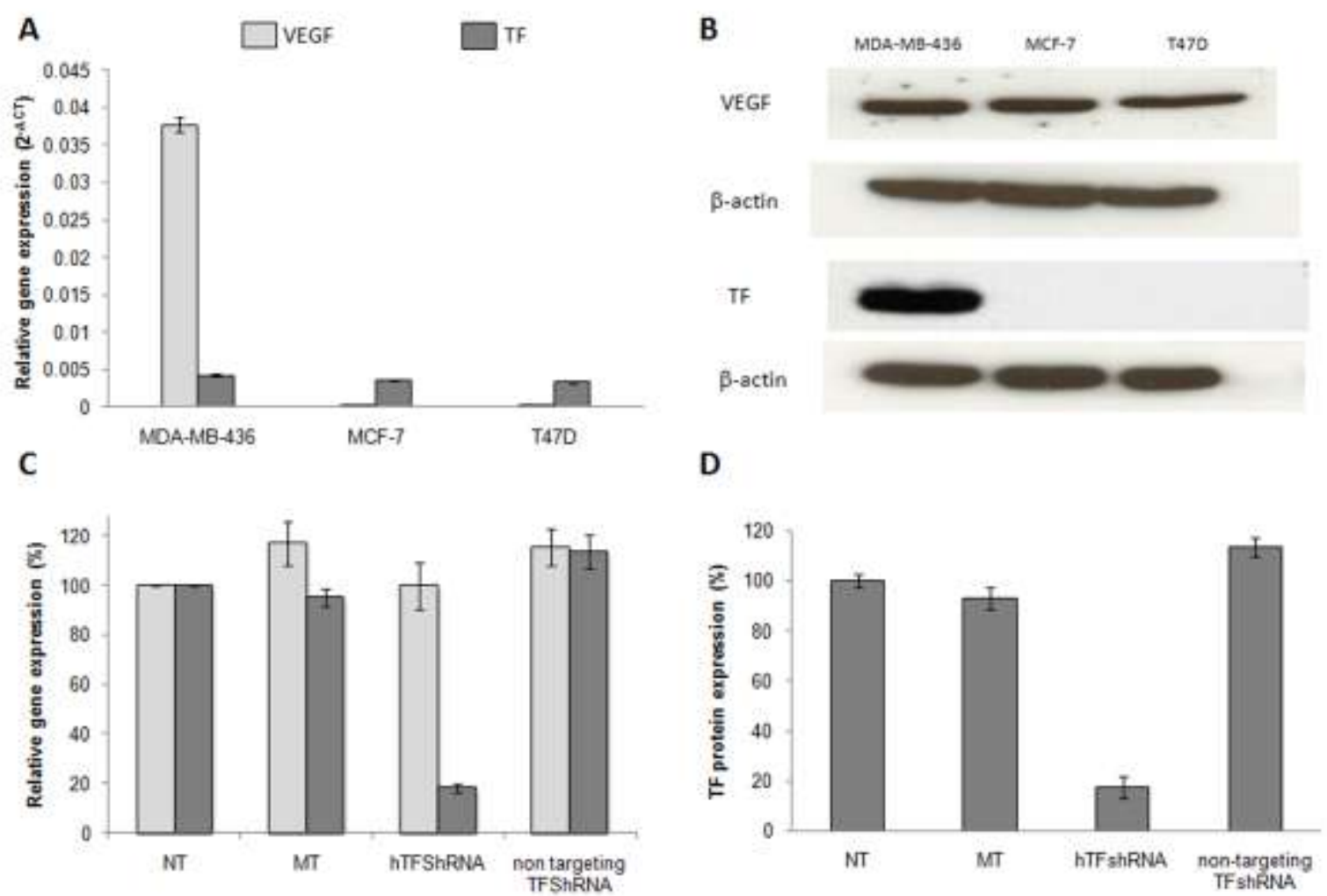

A

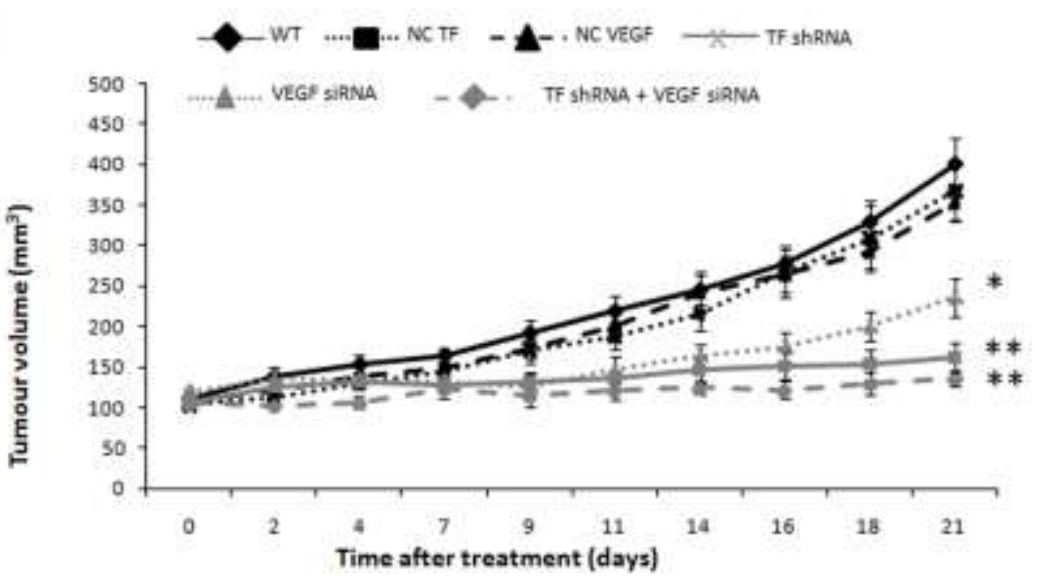



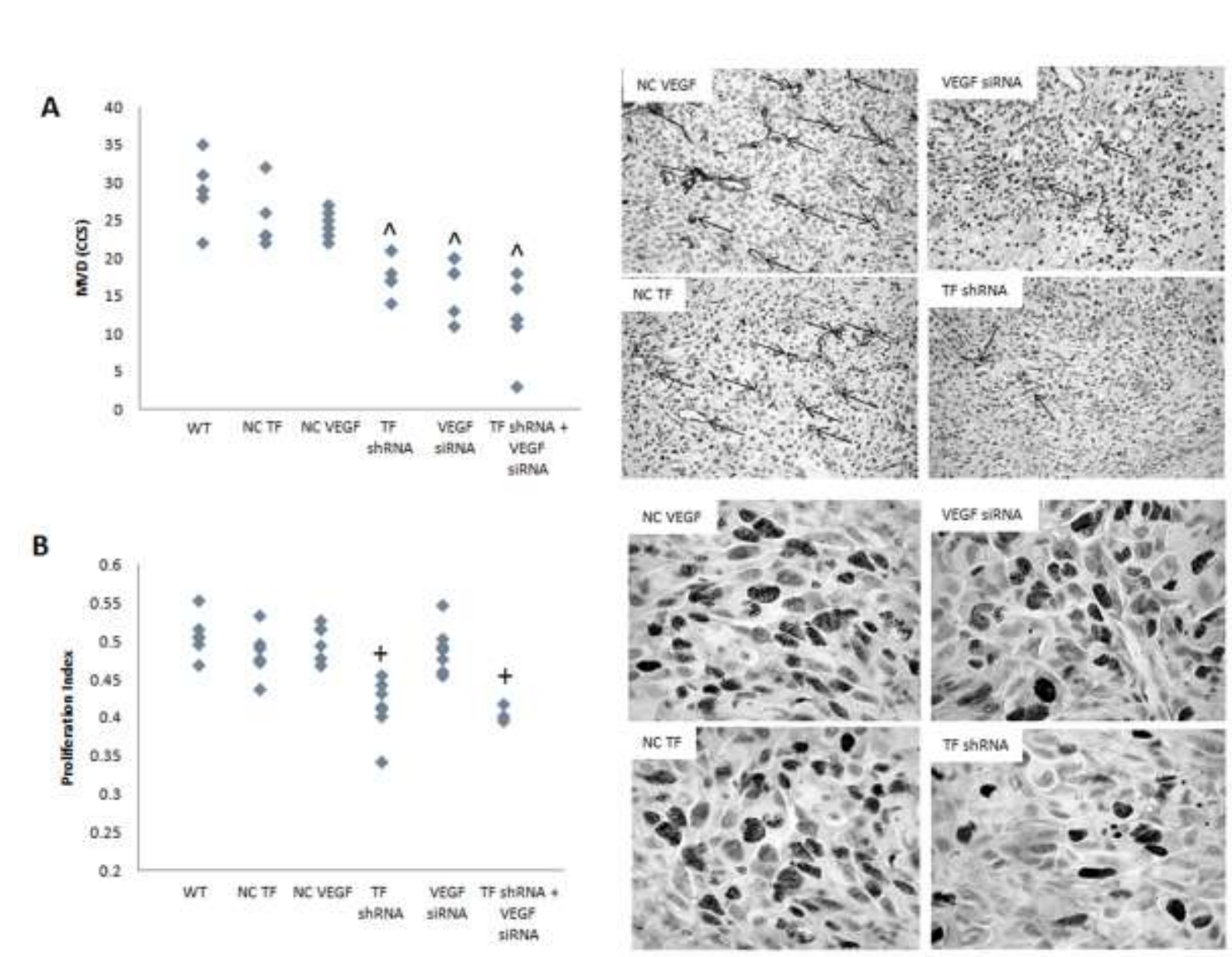